\title{
Commentary: More magic from Cleveland
}

\author{
Craig R. Smith, MD
}

\author{
From the Department of Surgery, Vagelos College of Physicians \& Surgeons of Columbia University, Columbia \\ University Irving Medical Center of New York Presbyterian Hospital, New York, NY. \\ No funding was received related to this article. \\ Disclosures: Author has nothing to disclose with regard to commercial support. \\ Received for publication June 1, 2019; accepted for publication June 3, 2019; available ahead of print July 3, 2019. \\ Address for reprints: Craig R. Smith, MD, Columbia University Irving Medical Center of New York Presbyterian \\ Hospital, Milstein Building 7-435, 177 Fort Washington Ave, New York, NY 10032 (E-mail: crs2@cumc. \\ columbia.edu) \\ J Thorac Cardiovasc Surg 2020;159:1679-80 \\ $0022-5223 / \$ 36.00$ \\ Copyright (C) 2019 by The American Association for Thoracic Surgery \\ https://doi.org/10.1016/j.jtcvs.2019.06.005
}

What should be done with a dilated ascending aorta, when the reason to operate is some other cardiac pathology? In this issue of the Journal, Idrees and colleagues ${ }^{1}$ attempt to answer this important question on the basis of a large experience at the Cleveland Clinic. Unfortunately, because all procedural decision making was left to the discretion of the many individual surgeons involved, we are immediately led down the rabbit hole of a complex propensity analysis that gave me migraine. I offer relief to those similarly afflicted: you need only know that in the 2 propensity-matched cohorts, early mortality was less than $1 \%$, with or without addressing the aorta. Stroke was well less than $2 \%$. Credit for these stunningly, astonishingly good results is appropriately given to "expert imaging, careful patient selection, refinement in surgical techniques, rescue from adverse events, and the recognized benefits that come with delivery of care by a dedicated multidisciplinary cardioaortic team." ${ }^{1}$ At the Cleveland Clinic, it appears that removal of a dilated aorta can be added to another kind of cardiac operation without penalty.

Is there a metamessage? Perhaps He-Who-Must-Not-Be Named looms in the background. "TAVoldemoRt" is rapidly invading all varieties of aortic valve disease on the basis of outstanding results in randomized trials of transcatheter aortic valve replacement (TAVR). Yet the results reported here are at least as good-might it be that clever patients who wand their way to the banks of the Cuyahoga are equally safe with surgery? Even (perish the thought!) the banks of the Hudson, the Charles, the Schuylkill? But centers of excellence cannot possibly treat everyone, and they cannot throw an invisibility cloak over the simplicity of transcatheter procedures.

It is important to note that Idrees and colleagues ${ }^{1}$ use prophylactic in their title, and they emphasize a "more proactive approach" in the text. Common adjectival synonyms for prophylactic include preventive and

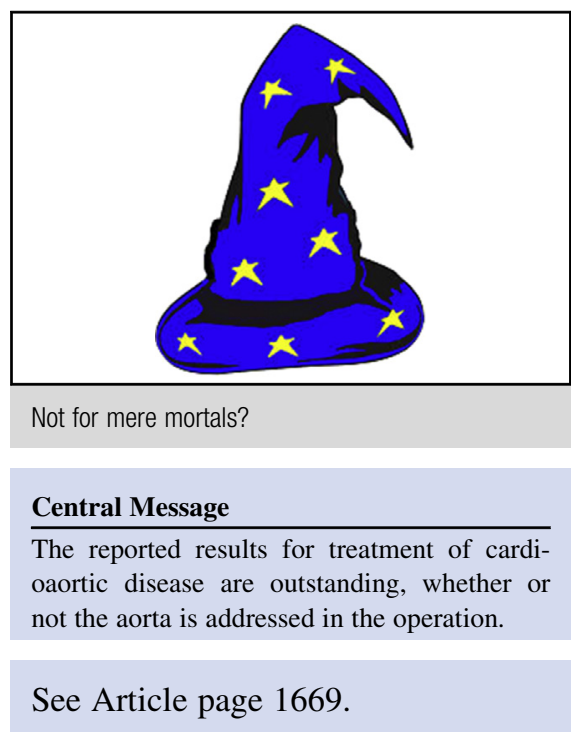

precautionary. Prophylactic certainly applies to patients with aortas larger than $4.5 \mathrm{~cm}$, because that size boundary in guidelines is based on evidence that removing aortas in that size range is an appropriate preventive precaution. Proactive is less a synonym than an embellishment of prophylactic, carrying as it does implications of boldness, dynamism, vision, and anticipatory action. I assume that Idrees and colleagues ${ }^{1}$ have adopted those nuances of proactive to support operating on aortas smaller than $4.5 \mathrm{~cm}$, outside current guidelines.

It may not be a coincidence that operating on patients with aortas smaller than indicated by current guidelines expands the definition of aortopathy, and aortopathy is currently a contraindication for TAVR. Note that the average aortic diameter in this series is 4.7 to $4.8 \mathrm{~cm}$ (see Table E1 in the article of Idrees and colleagues ${ }^{1}$ ). There is nothing prophylactic, proactive, or vaguely controversial about the decision making applied in that clear majority of patients in this series who had an aorta larger than $4.5 \mathrm{~cm}$. No data are presented separately, however, on patients with aortas falling below the $4.5-\mathrm{cm}$ threshold. The decision making of Idrees and colleagues ${ }^{1}$ outside current guidelines has thus been justified entirely by presenting overall outstanding results. Is that enough? Although it indirectly implies that risk is low, the benefit side of the equation can only be addressed by comparing long-term outcomes in the subgroup of patients with aortas smaller than $4.5 \mathrm{~cm}$ with those of similar patients who were left untreated. Opening new cardioaortic markets against TAVR may not work without evidence of 
benefit. To do so is not different from the proactive use of TAVR in asymptomatic mild aortic stenosis, which may be right around the corner.

\section{Reference}

1. Idrees JJ, Roselli EE, Blackstone EH, Lowry AM, Soltesz EG, Johnston DR, et al. Risk of adding prophylactic aorta replacement to a cardiac operation. J Thorac Cardiovasc Surg. 2020;159:1669-78.e10. 